\title{
New Insights into the Chemistry of Thiolate-Protected Palladium Nanoparticles
}

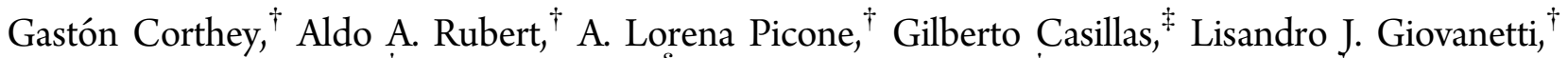 \\ José M. Ramallo-López, ${ }^{\dagger}$ Eugenia Zelaya, ${ }^{\S}$ Guillermo A. Benitez, ${ }^{\dagger}$ Félix G. Requejo, ${ }^{\dagger}$ \\ Miguel José-Yacamán, ${ }^{\dagger}$ Roberto C. Salvarezza, ${ }^{\dagger}$ and Mariano H. Fonticelli ${ }^{*}{ }^{\dagger}$ \\ ${ }^{\dagger}$ The Research Institute of Theoretical and Applied Physical Chemistry (INIFTA), National University of La Plata-CONICET,
Sucursal 4 Casilla de Correo 16, 1900 La Plata, Argentina
${ }^{\ddagger}$ Department of Physics and Astronomy, University of Texas at San Antonio, One UTSA Circle, San Antonio, Texas 78249, United
States
${ }^{\S}$ Bariloche Atomic Center, National Atomic Energy Commission-CONICET, Av. Bustillo 9500, 8400 S. C. de Bariloche, Río Negro,
Argentina
}

Supporting Information

ABSTRACT: This paper establishes the chemical nature of Pd nanoparticles protected by alkanethiolates that were prepared through a ligand placeexchange approach and the two-phase method, first developed for $\mathrm{Au}$ nanoparticles by Brust and Schiffrin. After 10 years since the first study on this kind of Pd nanoparticles was published, the surface composition of the particles is a matter of debate in the literature and it has not been unambiguously assessed. The nanoparticles were studied by means of several techniques: UV-visible spectroscopy, scanning transmission electron

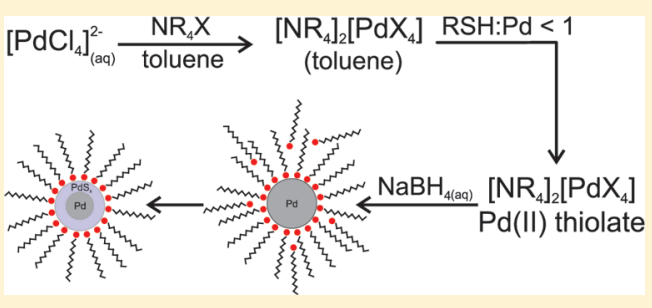
microscopy, Fourier-transform infrared spectroscopy, extended X-ray absorption fine structure, and X-ray photoelectron spectroscopy. The experimental data, obtained for the $3 \mathrm{~nm}$ diameter Pd particles, prepared by both synthetic routes, are consistent with nanoparticles composed by $\operatorname{Pd}(0)$ cores surrounded by a submonolayer of sulfide species, which are protected by alkanethiolates. Also, we unambiguously demonstrate that the chemical nature of these particles is very similar to that experimentally found for alkanethiolate-modified bulk Pd. The results from this paper are important not only for handling thiolate-protected Pd nanoparticles in catalysis and sensing, but also for the basic comprehension of metallic nanoparticles and the relation of their surface structure with the synthesis method.

\section{INTRODUCTION}

Noble metal nanoparticles (NPs) have recently attracted much attention due to their unique optical, electronic, and catalytic properties. The outstanding behavior of the different kinds of particles arises from their distinctive electronic properties, which are intimately related to their size and the chemical nature of their core and surface species. Among NPs of noble metals, those made of Au cores protected by thiolates represent the most studied and better understood systems, up to the point that, also when considering other metals, it is mandatory to refer to alkanethiolate-protected Au NPs. This fact resulted in some drawbacks in the comprehension upon the behavior of NPs of platinum group metals, as they behave differently than the Au ones in many aspects. This is particularly true regarding the surface chemistry of thiols on Pd. ${ }^{1}$ It is generally accepted that thiols form a thiolate bond when they are self-assembled on $\mathrm{Au}$ surfaces. ${ }^{2-6} \mathrm{On}$ the other hand, Pd planar surfaces are better described by considering a thin $\mathrm{PdS}_{x}$ surface, which is located between the bulk metal and an alkanethiolate selfassembled monolayer (SAM). ${ }^{1,7}$ Although this structure was proposed years ago by the Whitesides' group-and later studied in more detail in our laboratory ${ }^{1,8}$ - the complexity of
thiol/Pd system is still being underestimated. Certainly, even today it is simply described as a $\sqrt{3} \times \sqrt{3} \mathrm{R} 30^{\circ} n$ alkanethiolate SAM on $\operatorname{Pd}(111)$ surfaces. ${ }^{9-11}$ Also, when considering the surface of Pd NPs, the same lack of precision or incomplete description of their chemistry is observed in the literature. ${ }^{10,12-14}$ Recently, $\mathrm{Pd}$ particles (3.0 $\mathrm{nm}$ in size) prepared by the two-phase Brust-Schiffrin method ${ }^{3}$ in the presence of hexanethiol, for the application in hydrogen sensing and catalysis, where simply described as hexanethiolate-coated Pd monolayer-protected clusters. ${ }^{12}$ It was assumed that the replacement of amines by thiols is dominated by the strongest Pd-SR bond, without taking into account the possibility of the formation of a sulfide layer. On the other hand, Pd NPs have been alternatively described as palladium sulfide $\mathrm{NPs}^{15}$ or as a metallic Pd core capped by a dialkyl disulfide layer. ${ }^{16}$ The above examples indicate a clear need for a deeper comprehension of the interface formed when Pd NPs are protected by thiolderived species.

Received: February 15, 2012

Revised: $\quad$ March 27, 2012

Published: April 5, 2012 
Regarding the applications of these kinds of particles in catalysis, very recently, Pd NPs protected by several thiols were synthesized through a one-phase approach. Although intriguing differences in the crystallinity of the particles prepared at low and room temperature were reported, no further interpretation on the chemical bases was provided. ${ }^{17}$ They also emphasized that Suzuki cross-coupling reactions are generally carried out using relatively large amounts of expensive Pd salts or organopalladium complexes, and Pd NPs can be used to overcome this problem. Moreover, they successfully applied 11mercaptoundecanoic acid-covered Pd NPs as catalysts for coupling reactions and reused this material several times. In a recent paper, it was demonstrated that thiolate-protected Pd NPs are excellent catalyst for Heck reactions. ${ }^{18}$ In another interesting report, it was shown that alkanethiol coatings improve the selectivity of 1-epoxybutane formation from 1epoxy-3-butene on Pd catalysts from 11 to $94 \%$ at equivalent reaction conditions and conversions. It was found that, although sulfur species are generally considered to be indiscriminate catalyst poisons, the reaction rate for the desired product on a catalyst coated with a thiol was only slightly lower than that on an uncoated catalyst. However, the thiol/Pd surface was simply described as equivalent to $\mathrm{Au}$ modified by thiols. 10

All in all, provided the importance of Pd NPs due to their exceptional catalytic, sensing, and magnetic ${ }^{19}$ properties, an accurate interpretation of their electronic structure with the aim to understand the behavior of these NPs has as a prerequisite a correct description of the chemical composition and surface structure of these systems. In this regard, the approach made by Kornberg's and Häkkinen's groups is remarkable. ${ }^{5}$ They precisely determined the geometric structure of thiolateprotected $\mathrm{Au}$ NPs and, based on it, the electronic structure of these particles was described. ${ }^{20}$

The objective of the present study is to gain insight into the composition and geometric and electronic structures of Pd NPs protected by alkanethiols, which are approximately $3 \mathrm{~nm}$ in size. We have compared the chemical nature of the thiol-capping molecules on Pd NPs prepared by two different approaches: the NPs produced by the two-phase Brust-Schiffrin method and the ones obtained by ligand place-exchange of alkylamines by alkanethiols on previously synthesized alkylamine-protected Pd NPs. The results were contrasted with those obtained for alkanethiolate monolayer-protected Au NPs of comparable size and also with SAMs of alkanethiolates on extended planar Pd and $\mathrm{Au}$ surfaces. In this paper we demonstrate that the structure and composition of thiolate-protected Pd NPs is comparable to that found for extended surfaces. Metallic Pd cores are surrounded by a palladium sulfide with submonolayer coverage, while thiolate moieties protect them from sintering. This structure is found both for Pd NPs prepared by the Brust-Schiffrin method and those produced by ligand placeexchange.

\section{EXPERIMENTAL SECTION}

2.1. Synthesis of the Nanoparticles. Dodecanethiolateprotected Pd NPs were synthesized by the Brust-Shriffrin method $^{3}$ or by ligand place-exchange of dodecylamineprotected Pd NPs, prepared by the Leff method. ${ }^{21}$ Details on the synthesis of the nanoparticles are given in the Supporting Information.

2.2. UV/Visible Absorption Spectroscopy (UV/Vis). $\mathrm{UV} /$ vis spectra were recorded with a Perkin-Elmer Lambda
35 spectrometer, equipped with a double beam. Hexane or toluene was used as reference.

2.3. Fourier-Transform Infrared Spectroscopy (FTIR). FTIR spectra were recorded on a nitrogen-purged Varian 660 spectrometer equipped with a DTGS detector. A thick film of the sample was prepared on a $\mathrm{KBr}$ window by drop-casting a toluene solution of the NPs, which was further dried under nitrogen. The spectra were acquired in the transmission mode with a spectral resolution of $4 \mathrm{~cm}^{-1}$ accumulating 128 scans. Additionally, the baselines of the spectra were corrected using the Varian FTIR spectrometer software.

2.4. Scanning Transmission Electron Microscopy (STEM). STEM was carried out with a JEOL JEM-ARM200F aberration-corrected microscope, operating at $200 \mathrm{kV}$, equipped with a Schottky FEG, a hexapole spherical aberration $\left(C_{s}\right)$ probe corrector $(\mathrm{CEOS} \mathrm{GmbH})$, and a high-angle annular dark field (HAADF) detector. The probe size used for acquiring the HAADF images was $0.095 \mathrm{~nm}$. The alignment of the microscope was verified through the CESCOR software. A focus/tilt tableau was acquired measuring defocus and 2-fold astigmatism as a function of both radial and azimuthal tilt angles. HAADF-STEM images were acquired with a camera length of $120 \mathrm{~mm}$ and a collection angle of 33-125 mrad. Nanoparticle suspension was drop-cast on an amorphous carbon-covered copper grid. Details on the size distribution of the particles are included in the Supporting Information.

2.5. Extended X-ray Absorption Fine Structure (EXAFS). EXAFS experiments at the Pd K edge $(24350 \mathrm{eV})$ were performed using a RIGAKU R-XAS Looper in-house spectrometer in transmission mode. Ionization chambers filled with $\mathrm{Xe}$ were used to measure the incident radiation and a solid state detector to measure the transmitted intensity. Homogeneous dry samples of Pd@SC12 NPs were mounted on an acrylic sample holder. The thickness of the sample provided an X-ray absorption jump at the Pd K edge of approximately 0.75 . The energy calibration and the determination of the $S_{0}=0.56$ \pm 0.02 were done using a metallic Pd foil.

2.6. X-ray Photoelectron Spectroscopy (XPS). The samples were characterized by XPS using both a conventional $\mathrm{X}$-ray source and synchrotron radiation. In the laboratory, a $\mathrm{Mg}$ $\mathrm{K} \alpha$ source (XR50, Specs $\mathrm{GmbH}$ ) and a hemispherical electron energy analyzer (PHOIBOS 100, Specs GmbH) were used. A two-point calibration of the energy scale was performed using sputtered, cleaned $\mathrm{Au}\left[\mathrm{Au} \mathrm{4 \textrm {f } _ { 7 / 2 }}\right.$, binding energy $(\mathrm{BE})=84.00$ $\mathrm{eV}]$ and copper $\left(\mathrm{Cu} 2 \mathrm{p}_{3 / 2}, \mathrm{BE}=932.67 \mathrm{eV}\right)$ samples. The base pressure inside the ultrahigh vacuum (UHV) chamber was below $10^{-9}$ mbar. XPS was also performed at the SGM beamline of the Laboratorio Nacional do Luz Síncrotron (LNLS), Campinas, Brazil. This beamline is equipped with a Spherical Grating Monochromator, which allows working in the range of $250-1000 \mathrm{eV}$. The endstation is composed by an UHV chamber (base pressure $=10^{-8} \mathrm{mbar}$ ) with a hemispherical electron energy analyzer (PHOIBOS 150, Specs $\mathrm{GmbH})$. The energy of the incident photons was set to $250 \mathrm{eV}$. For spectra deconvolution of the S $2 p$ region, a Shirley-type background and a Gaussian-Lorentzian function were used. The full width at half-maximum (fwhm) was fixed at $1.1 \mathrm{eV}$ in the case of $1253.6 \mathrm{eV}$ of incident energy and $0.8-0.9 \mathrm{eV}$ for $250 \mathrm{eV}$. The spin-orbit doublet separation of $\mathrm{S} 2 \mathrm{p}$ signal was set to $1.2 \mathrm{eV}$. The BEs and peak areas were optimized to achieve the best fit. S:Pd and C:S atomic ratios were estimated by the measurement of the areas of $\mathrm{Pd} 3 \mathrm{~d}, \mathrm{~S} 2 \mathrm{p}$, and $\mathrm{C} 1 \mathrm{~s}$ signals, corrected by the atomic ionization cross sections at the 


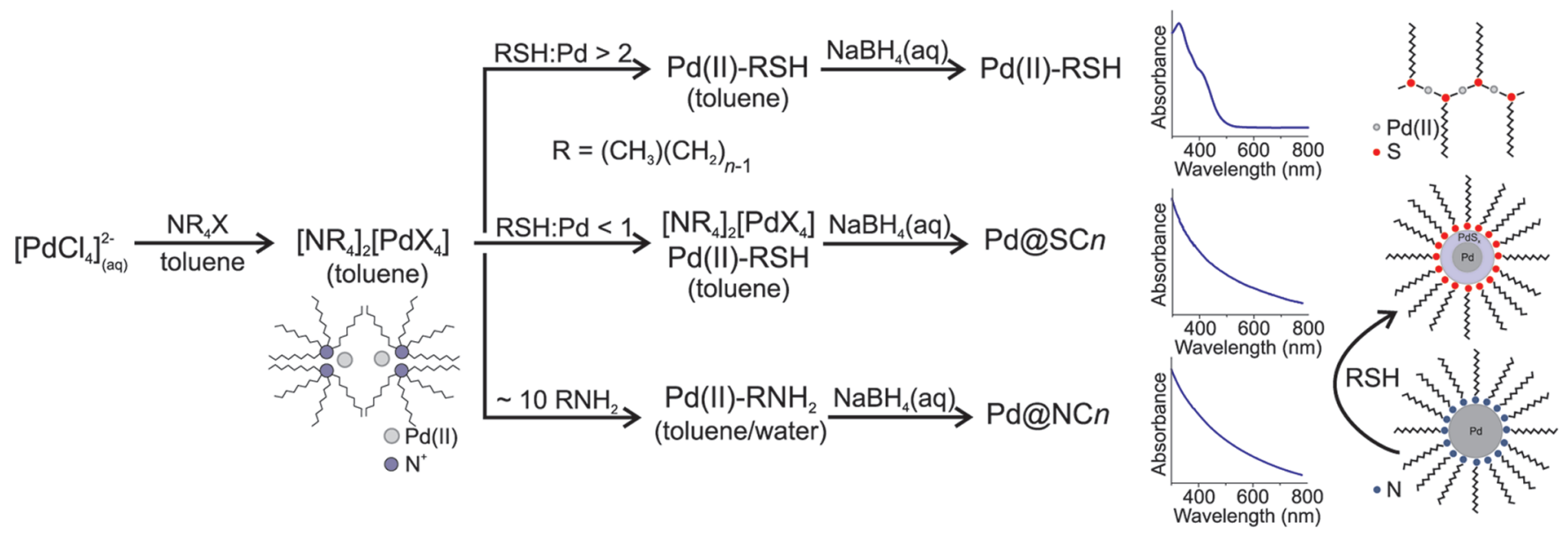

Figure 1. Scheme of the different routes for the synthesis of Pd NPs and Pd(II) thiolate complexes. The UV-vis absorption spectra of different products are also shown. For the systems studied in this paper, $\mathrm{SC} n=\mathrm{SC} 12$ and $\mathrm{NC} n=\mathrm{NC} 12$. The drawings are only schematic representations of the products or intermediates.

corresponding X-ray energies. ${ }^{22}$ The dried NPs were suspended in hexane and drop-cast on graphite or a platinum foil substrate and then dried before their introduction at the main chamber. Valence-band region was measured only in samples deposited on graphite and C:S atomic ratio only in samples deposited on platinum foil.

\section{RESULTS AND DISCUSSION}

The first route employed for the synthesis of dodecanethiolateprotected Pd NPs was the two-phase method developed by Brust and Schiffrin for the synthesis of thiolate monolayerprotected $\mathrm{Au} \mathrm{NPs}{ }^{3}{ }^{3}$ This method was used for the first time with Pd by Chen et al. ${ }^{23}$ and it was later studied in more detail by Zamborini et al. ${ }^{24}$ As represented in the scheme of Figure 1, $\mathrm{Pd}(\mathrm{II})$ species that form halide complexes $\left(\left[\mathrm{PdCl}_{4}\right]^{2-}\right)$ are transferred from the aqueous solution to the organic phase by a quaternary ammonium salt $\left(\mathrm{NR}_{4} \mathrm{X}\right)$. Metal ions might reside inside inverse micelles of the ammonium salt in the organic solvent. ${ }^{25}$ As bromide anions can be exchanged with chlorides in the coordination sphere of $\mathrm{Pd}(\mathrm{II})$, the Pd complexes in the organic phase are simply called $\left[\mathrm{PdX}_{4}\right]^{2-}$. Contrary to what happens when this protocol is carried out for the synthesis of $\mathrm{Au} \mathrm{NPs},{ }^{26}$ the addition of thiols (RSH) does not drive the reduction of the metallic species; i.e., palladium remains as Pd(II) after thiol addition. Furthermore, while recent experimental data have shown that ion-pairs of tetraalkylammonium and $\mathrm{Au}(\mathrm{I})$-halide complexes $\left(\left[\mathrm{NR}_{4}\right]\left[\mathrm{AuX}_{2}\right]\right)$ are the real precursors in the two-phase method for the synthesis of $\mathrm{Au}$ $\mathrm{NPs}^{26} \mathrm{Pd}(\mathrm{II})$-thiol complexes are formed when thiols are added to Pd(II) species dissolved in toluene. ${ }^{24}$ However, as the thiol amount used in the present study (0.5:1 thiol:Pd molar ratio) is lower than that needed to completely form the $\mathrm{Pd}(\mathrm{II})$-thiolate complexes, also $\left[\mathrm{PdX}_{4}\right]^{2-}$ species are present in the organic phase when the reducing agent, sodium borohydride, is added (see Supporting Information). When this happens, the reduction of $\mathrm{Pd}(\mathrm{II})$ species to $\operatorname{Pd}(0)$ is produced and dodecanethiolate-protected Pd NPs (Pd@SC12) are obtained. The attempts to form Pd NPs starting with thiol:Pd molar ratios higher than 1:1 have failed (see Supporting Information). ${ }^{24,27}$ The UV-vis absorption spectroscopy data (Figure 1) correlate very well with those previously reported for Pd NPs. ${ }^{13,23,24}$ The absence of peaks in the 300-500 $\mathrm{nm}$ domain, which are related to $\mathrm{Pd}(\mathrm{II})-$ thiolates, $^{24,27,28}$ indicates that no significant amount of these species is present in Pd NPs. ${ }^{13,23,24}$ It was not possible to prepare propanethiolate-protected $\mathrm{Pd}$ nanoparticles because of the short hydrocarbon chain length of the thiols.

The second route employed was the ligand place-exchange of alkylamines $\left(\mathrm{RNH}_{2}\right)$ by alkanethiols on previously synthesized dodecylamine-protected Pd NPs (Pd@NC12). To prepare these particles, the Leff method was followed. ${ }^{21}$ These particles are commonly described as a $\operatorname{Pd}(0)$ core protected by alkylamine molecules, ${ }^{12,29}$ as depicted in Figure 1. However, since the interaction of alkylamines with Pd is not as strong as with thiols, these NPs are more susceptible than Pd@SC12 ones to being oxidized by the oxygen present in the reaction media. Therefore, some amount of palladium oxide could be present in these particles.

After rinsing Pd@NC12 NPs, they were placed in contact with dodecanethiol (thiol:Pd molar ratio $\sim 1: 1$ ) in toluene overnight to accomplish the ligand place-exchange. After rinsing these particles with ethanol, Pd NPs covered by a mixture of dodecylamine and dodecanethiol were obtained (Pd@NC12-SC12).

Figure 2 presents STEM images, recorded using a HAADF detector (HAADF-STEM), of Pd@NC12 and Pd@SC12 NPs. In this configuration-also called Z-contrast imaging-the intensity of the signal is approximately proportional to the square of the atomic number $\left(\sim Z^{2}\right)$ of the elements in the specimen and its thickness. ${ }^{30}$ Consequently, Pd atoms from the NPs appear with white contrast on the image surrounded by the almost black background corresponding to the amorphous carbon support. As insets in Figure 2, high-resolution HAADFSTEM images of Pd@SC12 and Pd@NC12 are also shown. The results observed in these images disagree with the model proposed by Sun et al., for Pd@SC12 NPs prepared in a similar way. ${ }^{31}$ In that paper, the authors proposed that $\operatorname{Pd}(0)$ clusters were immersed in a palladium sulfide phase. If this were the case, the background of the HAADF-STEM images should be brighter than in our micrographs, due to the presence of the Pd atoms surrounding the NPs. Thus, we can affirm that Pd atoms are constrained into a well-defined size on the order of $\sim 3 \mathrm{~nm}$, rather than dispersed in an extended palladium sulfide phase.

The high-resolution HAADF-STEM images also show a clear difference in the crystallinity of the particles. While Pd@NC12 NPs present a crystalline structure, it was not possible to 

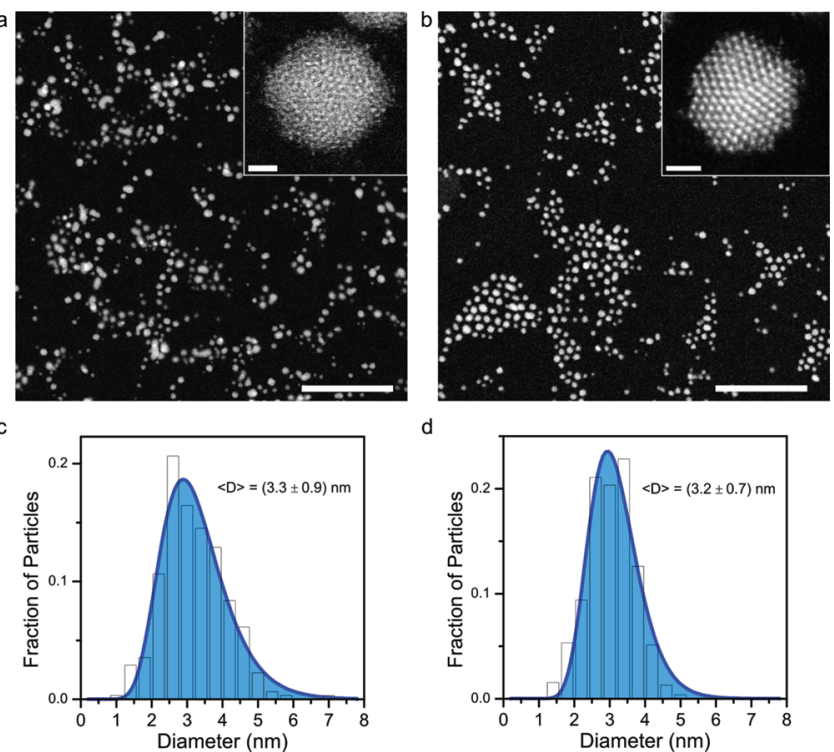

Figure 2. Representative HAADF-STEM images of (a) Pd@SC12 NPs and (b) Pd@NC12 NPs. Scale bars = $50 \mathrm{~nm}$. The insets show high-resolution images of the NPs (scale bars $=1 \mathrm{~nm}$ ). Particle size distribution histograms and their log-normal fit for (c) Pd@SC12 NPs and (d) Pd@NC12 NPs.

distinguish lattice fringes in any of the images of Pd@SC12 NPs for any defocus value. This is clear evidence that the crystalline structure of the particle is largely affected by the capping agent: the strong $\mathrm{Pd}$-thiolate interaction or the incorporation of sulfur, as sulfide, on the nanoparticle surface could be responsible for these distortions. Similar results were already obtained, but the physical origin of those images remains unclear. ${ }^{17,32,33}$ A detailed study on these phenomena, supported by image simulations, will be reported in a future work.

FTIR spectroscopy was used to verify the presence of the dodecanethiol-derived species as protecting agents of the Pd@ SC12 NPs. The position of the methylene symmetric $\left(\mathrm{d}^{+}\right)$and antisymmetric $\left(\mathrm{d}^{-}\right) \mathrm{CH}_{2}$ stretching vibrations $\left(\nu_{\mathrm{d}+}=2850 \mathrm{~cm}^{-1}\right.$ and $\nu_{\mathrm{d}-}=2921 \mathrm{~cm}^{-1}$ ) in the FTIR spectrum (Figure 3) reveals that the alkyl chains are present and extended in a trans zigzag conformation. ${ }^{34}$ The absence of an absorption peak in the $\nu(\mathrm{S}-$ $\mathrm{H})$ region (inset of Figure 3), which appears at $2575 \mathrm{~cm}^{-1}$ for free dodecanethiol, indicates the breakage of the $\mathrm{S}-\mathrm{H}$ bond of the dodecanethiol. ${ }^{35}$ In the low wavenumber region of the

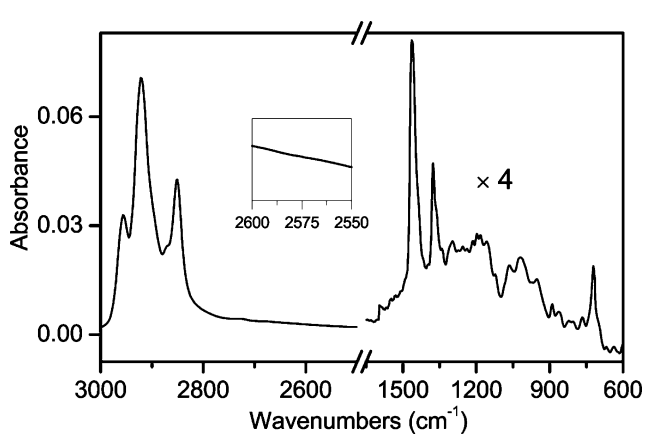

Figure 3. FTIR spectrum of Pd@SC12 NPs. The absorbance in the region between 1650 and $600 \mathrm{~cm}^{-1}$ was multiplied by a factor of 4 . In the inset, the region near $2575 \mathrm{~cm}^{-1}$ is amplified to verify the absence of the peak corresponding to $\nu(\mathrm{S}-\mathrm{H})$. spectrum, the peak at $720 \mathrm{~cm}^{-1}\left(\mathrm{P}_{1}\right)$ is the principal band of the rocking progression. ${ }^{34}$ As reported for thiolate-covered $\mathrm{Au}$ nanoparticles, ${ }^{36}$ the $\mathrm{C}-\mathrm{S}$ stretches adjacent to trans-methylene units $\nu(\mathrm{C}-\mathrm{S})_{\mathrm{T}}$ appear as a significantly intense shoulder at 700 $\mathrm{cm}^{-1} \cdot{ }^{36,37}$ On the other hand, the gauche band $\nu(\mathrm{C}-\mathrm{S})_{\mathrm{G}}$ was undetectable (i.e., the uncertainty associated with the background subtraction has a magnitude comparable to the intensity of the small peaks observed for wavenumbers smaller than 700 $\left.\mathrm{cm}^{-1}\right)$. Thus, we can conclude that the number of adsorbates with the $\mathrm{C}-\mathrm{C}$ bond adjacent to the $\mathrm{C}-\mathrm{S}$ in gauche conformation is relatively low. Contrary to our results, a $100 \%$ gauche conformation, which was interpreted considering dioctyl disulfide as capping species, was found for octanethiolprotected Pd NPs. ${ }^{16}$ See the Supporting Information for a detailed analysis of the FTIR data.

In the following, X-ray absorption and photoelectron spectroscopies will give valuable information with regard to the structure, chemical composition, and electronic properties of Pd NPs.

In order to carry out the EXAFS analysis, a dry sample of Pd@SC12 NPs was studied at the Pd K-edge to determine the radial distribution of the atoms, i.e., the average coordination number and Pd-bond distances. EXAFS spectrum of bulk Pd was also recorded for comparison and calibration purposes. The Fourier Transform (FT) of the EXAFS data corresponding to Pd NPs is shown in Figure 4. This spectrum exhibits two main

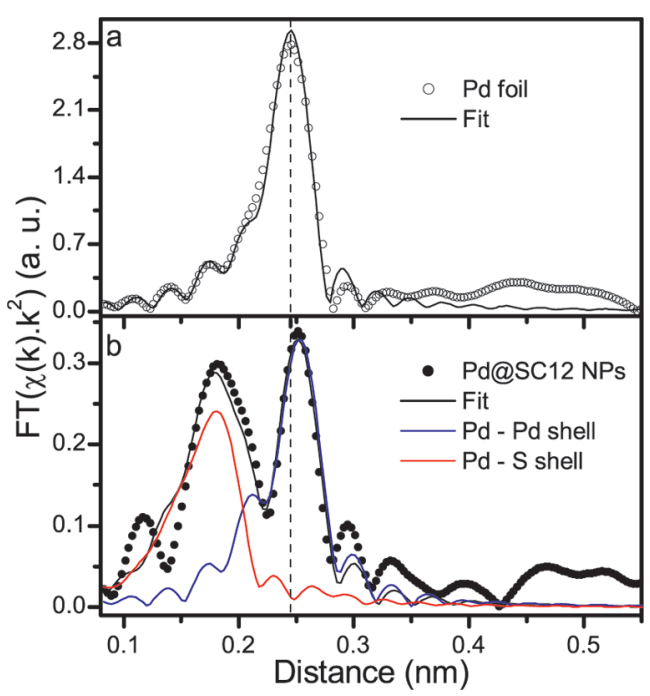

Figure 4. Experimental Fourier transform and corresponding fits of the EXAFS signal for (a) Pd foil and (b) Pd@SC12 NPs sample.

contributions between 0.13 and $0.28 \mathrm{~nm}$ (uncorrected for the photoelectron phase shifts). The presence of $\mathrm{Pd}-\mathrm{Pd}$ contribution with a bond distance longer than that of the bulk Pd can be determined by comparison with the FT of the Pd foil signal. ${ }^{19,31,38}$ We attribute the contribution in the FT between 0.13 and $0.21 \mathrm{~nm}$ to the presence of $\mathrm{Pd}-\mathrm{S}$ coordination shells. ${ }^{15,19,31,38}$ To analyze the first nearest neighbor region we did a nonlinear curve fit using the IFEFFIT package, ${ }^{39,40}$ integrated into the ATHENA and ARTEMIS user interfaces. The fitted parameters for each coordination shell proposed in the model were the average coordination number $(N)$, path length $(d)$, correction to the threshold energy $\left(\Delta E_{0}\right)$, and Debye-Waller factor $\left(\sigma^{2}\right)$. To perform the fitting, two different shells were proposed, one to take into account the 
$\mathrm{Pd}-\mathrm{Pd}$ contribution and the other the $\mathrm{Pd}-\mathrm{S}$ shell. These contributions were calculated using the FEFF $\operatorname{code}^{41}$ from the crystallographic structure of metallic Pd and palladium sulfide. Since sulfur from the sample suffered from radiation-induced damage during the measurements (generation of high oxidation state sulfur species), the deconvolution of this signal into contributions of different $\mathrm{Pd}-\mathrm{S}$ distances coming from different sulfur species might not be reliable. Accordingly, the $\mathrm{Pd}-\mathrm{S}$ shell was fitted considering only one species, although it can be composed by different compounds. The results of the analysis of the EXAFS data shown in Figure 4 are reported in Table 1.

Table 1. Structural Parameters of Pd@SC12 NPs Obtained by EXAFS ${ }^{a}$

\begin{tabular}{lcccc} 
& \multicolumn{2}{c}{ Pd@SC12 NPs } & & Pd foil: \\
\cline { 2 - 3 } \cline { 5 - 5 }$N$ & Pd-Pd shell & Pd-S shell & & Pd-Pd shell \\
$\Delta E_{0}(\mathrm{eV})$ & $1.8 \pm 0.6$ & $1.3 \pm 0.5$ & & 12 \\
$\sigma^{2}\left(10^{-5} \mathrm{~nm}^{2}\right)$ & $6 \pm 2$ & $7 \pm 2$ & & $-1 \pm 2$ \\
$d(\mathrm{~nm})$ & $0.275 \pm 0.001$ & $0.229 \pm 0.003$ & & $0.273 \pm 0.001$
\end{tabular}

${ }^{a}$ Average coordination number $(N)$, path distance $(d)$, threshold energy correction $\left(\Delta E_{0}\right)$, and Debye-Waller exponent $\left(\sigma^{2}\right)$.

The small $N$ value $(1.8 \pm 0.6)$ for the Pd-Pd contribution cannot be only explained by the formation of small Pd NPs. Instead of that, another phase with no Pd as first neighbors is needed in order to explain the low $N$. This is due to the fact that this parameter represents an average over all $\mathrm{Pd}$ atoms present in the sample. If a fraction of Pd atoms is forming a structure in which they are bonded to a different type of atom, they will contribute with zero to the $\mathrm{Pd}-\mathrm{Pd}$ average coordination number. The $\mathrm{Pd}-\mathrm{Pd}$ distance obtained in the fitting of Pd@SC12 NPs $(0.275 \pm 0.001 \mathrm{~nm})$ is larger than that obtained for bulk Pd $(0.273 \pm 0.001 \mathrm{~nm})$. Similar results for $\mathrm{Pd}-\mathrm{Pd}$ distances in Pd NPs capped with thiols were obtained earlier. $^{31,38}$

The EXAFS data agree with previous studies. However, the low $N$ was interpreted by two models. The first one proposes $\operatorname{Pd}(0)$ clusters immersed in a palladium sulfide phase, which we discarded on the basis of our HAADF-STEM analysis. ${ }^{31}$ The second one proposes a Pd-core $\mathrm{PdS}_{x}$-shell structure covered by thiolate moieties, in analogy with the planar Pd surfaces modified with thiols. ${ }^{19,38}$ However, the EXAFS data gave no further details with regard to the chemistry of the $\mathrm{S} / \mathrm{Pd}$ interface or the processes that occur during the formation of NPs.

In order to address the surface chemical composition of Pd@ SC12 NPs, we will discuss XPS data of this material, in relation with results obtained for dodecanethiolate-protected Au NPs (Au@SC12, prepared as explained in the Supporting Information), Pd@NC12, Pd@NC12-SC12 NPs, and dodecanethiolate SAMs on extended $\mathrm{Pd}$ and $\mathrm{Au}$ surfaces.

XPS spectra for the Pd@SC12 NPs show a broad S 2p signal at $\sim 162.5 \mathrm{eV}$ (Figure 5a). The analysis of the $\mathrm{S} 2 \mathrm{p}$ and $\mathrm{Pd} 3 \mathrm{~d}$ signals indicate that the total sulfur to palladium ratio $(\mathrm{S}: \mathrm{Pd}=$ $0.7 \pm 0.1)$ is twice the value found for the S:Au ratio in $\mathrm{Au} @$ SC12 NPs of comparable size. On the other hand, the same factor is found in the $(\mathrm{S}: \mathrm{Pd}) /(\mathrm{S}: \mathrm{Au})$ ratio derived from XPS $\mathrm{S}$ $2 p$ data of alkanethiolate SAMs on $\operatorname{Pd}(111)^{7,8}$ and $\operatorname{Au}(111){ }^{2}$ This value is clear evidence that there is a sulfur excess in the Pd@SC12 NPs surfaces in relation to that expected in systems where the capping species are alkanethiolates. A similar conclusion can be derived from the thermogravimetric analysis reported by Zamborini et al., who found a bigger organic content than that predicted on the basis of a simple thiolate adsorption model. $^{24}$ On the basis of these results we can immediately discard simple thiolate-Pd interface models reported in recent years for this system. ${ }^{10,12,13}$

The fitting of the $S 2 p$ peak yields two main components at 162.1 and $162.9 \mathrm{eV}$ and a small one at $164.1 \mathrm{eV}$ (Figure 5a). The assignment of the components was done following the work by the Whitesides' group for alkanethiolate adlayers on bulk Pd: thiolates (162.9 eV component) are placed on sites of a diluted sulfide layer (162.1 eV component) adsorbed on the nanoparticle surface. ${ }^{1,7,8}$ The assignment of the small $164.1 \mathrm{eV}$ component is more complicated and it could correspond to physisorbed disulfide molecules ${ }^{7}$ or physisorbed alkanethiols. ${ }^{42}$ Note that our results disagree with the data reported for $n$ octadecyl mercaptan-protected Pd NPs prepared by the twophase method, for which binding energies were found to range from 161.4 to $161.7 \mathrm{eV}^{43}$

For the sake of comparison, the $S 2 p$ components for dodecanethiolate-modified extended planar $\mathrm{Pd}$ surfaces are presented (Figure $5 \mathrm{~b}$ ). It is evident that the components in the spectra of planar and nanoparticle Pd surfaces are quite similar and clearly different from that corresponding to Au@SC12 NPs (Figure 5d) and previously reported data for $\mathrm{Au}(111)$ surfaces. $^{2}$ These results evidence that sulfur species present on both $\mathrm{Pd}$ surfaces, planar and $3 \mathrm{~nm}$ NPs, are probably of the same nature. If dialkyl disulfides were the main ligands, as previously
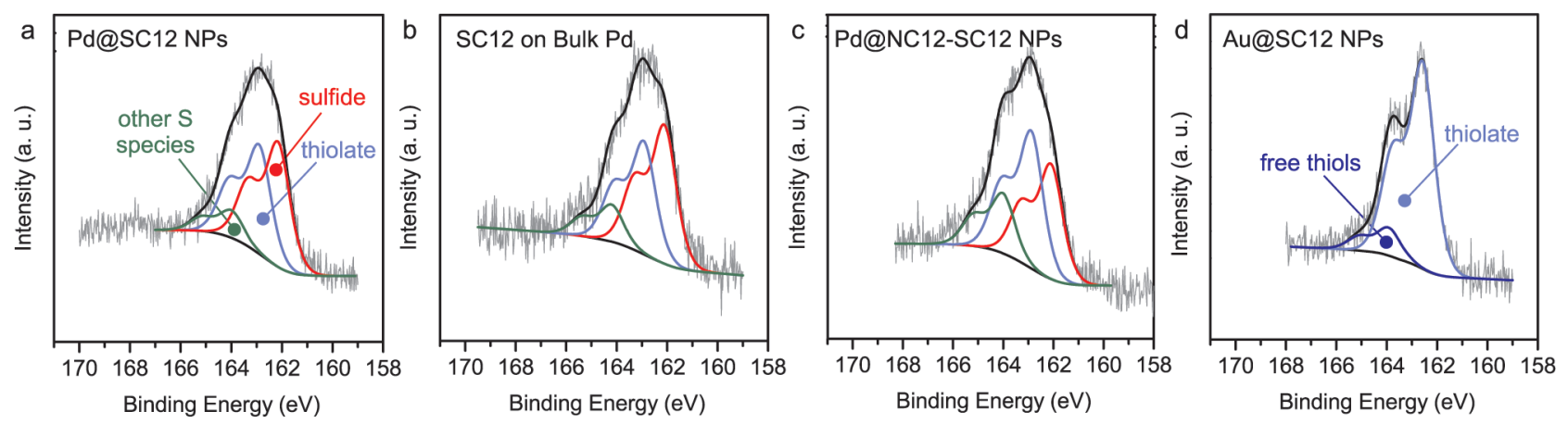

Figure 5. The S 2p spectra of different systems are shown for comparison: (a) Pd@SC12 NPs prepared by two-phase Brust-Schiffrin method, (b) dodecanethiol adsorbed on bulk Pd, (c) Pd@NC12-SC12 NPs prepared by ligand place-exchange of Pd@NC12 NPs with dodecanethiol, and (d) Au@SC12 NPs prepared by two-phase Brust-Schiffrin method. 
proposed, ${ }^{16}$ the $163-164 \mathrm{eV}$ component should dominate the S 2p signal, which is clearly not the case in Pd@SC12 NPs (Figure 5a).

The C:S atomic ratio obtained is smaller than that expected for NPs capped only by thiolate moieties, in concordance with the presence of a mixed sulfide/thiolate adlayer (see Supporting Information).

Dodecanethiolate-protected Pd NPs prepared by ligand place-exchange of Pd@NC12 NPs were also studied by XPS. The alkylamines were partially exchanged by dodecanethiol molecules, resulting in particles with a mixed capping agent (Pd@NC12-SC12 NPs), as revealed by XPS, which evidenced some amount of nitrogen after the ligand place-exchange. In Figure 5c, it can be observed that these NPs have a composition very similar to that of Pd@SC12 NPs, prepared by the twophase method. The quantitative data is presented in Table 2.

Table 2. XPS Data for Different Dodecanethiolate-Covered Pd Surfaces ${ }^{a}$

\begin{tabular}{lccc} 
& Bulk Pd & $\begin{array}{c}\text { Pd@SC12 } \\
\text { NPS }\end{array}$ & $\begin{array}{c}\text { Pd@NC12-SC12 } \\
\text { NPs }\end{array}$ \\
\hline sulfide & $48 \pm 3$ & $46 \pm 2$ & $37 \pm 3$ \\
$\%$ thiolate & $39 \pm 4$ & $42 \pm 3$ & $44 \pm 4$ \\
$\begin{array}{c}\text { \%-S-S-, } S_{n} \text {, or physisorbed } \\
\text { thiols }\end{array}$ & $13 \pm 2$ & $12 \pm 3$ & $19 \pm 6$ \\
\hline
\end{tabular}

${ }^{a}$ Relative contribution of the different components of the $S 2 p$ signal for dodecanethiol SAM on bulk Pd, Pd NPs prepared by the BrustSchiffrin method (Pd@SC12), and Pd NPs prepared by ligand placeexchange of Pd@NC12 NPs (Pd@NC12-SC12 NPs).

Although the total S:Pd molar ratio was lower compared to that of Pd@SC12 NPs, due to an incomplete exchange of the ligands, the contributions to the $S 2 p$ peak show the presence of sulfide in these NPs. This makes evident the S-C bond breakage by Pd core upon thiol adsorption. Even though these two routes to obtain thiolate-protected NPs are completely different, the final composition is very similar.

In order to get insight into the distribution of the different sulfur species in the nanoparticles, we carried out highresolution XPS measurements with X-rays of lower energies. The $250 \mathrm{eV}$ synchrotron light used for the analysis of the S $2 \mathrm{p}$ signal produces photoelectrons of markedly lower kinetic energies than those produced in our laboratory with a $\mathrm{Mg} \mathrm{K} \alpha$ source. The measurements at $250 \mathrm{eV}$ allowed sensitivity to the composition of the surface rather than to the whole nanoparticle. In this regard, it is interesting to note that the inelastic mean free path of the $S 2 p$ photoelectrons emitted due to the incidence $\mathrm{Mg} \mathrm{K} \alpha$ radiation $(1253.6 \mathrm{eV})$ in a metallic phase is about $2.5 \mathrm{~nm}$, while for those produced by an incident photon energy of $250 \mathrm{eV}$ it is estimated to be $0.5 \mathrm{~nm} .{ }^{44}$ The deconvolution of the spectrum taken with $250 \mathrm{eV}$ (see Supporting Information) showed the same thiolate to sulfide area ratio $(\sim 1)$ that was found with the $\mathrm{Mg} \mathrm{K} \alpha$ source $(\sim 0.9)$, within the experimental error. If sulfide was homogeneously distributed in the cores of the Pd@SC12 NPs, its relative contribution to the $S 2 p$ signal should be smaller in the case $250 \mathrm{eV}$ incident energy. Thus, we can infer that thiolate and sulfide species are both at the surface of the NPs. On the basis of it, we can discard the idea of sulfide homogeneously distributed in the cores of the nanoparticles.

In summary, independent of the route used to produce thiolate-protected Pd NPs and the nature of the surface (extended planar or NPs), comparable amounts of thiolate and sulfide species are found as components of the systems. Additionally, the sulfur species are located in the surface of the nanoparticles. It is important to note that this last conclusion could not be reached from EXAFS or conventional XPS.

The analysis of the Pd $3 \mathrm{~d}$ signal is also important in order to understand the nature of sulfur species on $\mathrm{Pd}$ nanoparticle surface. When compared to clean Pd surface, the samples prepared in the presence of thiols exhibit a significant shift toward greater binding energies, indicating partial Pd oxidation, which has been related to the presence of $\operatorname{PdS}_{x}$ at the interface. ${ }^{8,33}$ However, as was already stated by Cook et al., ${ }^{45}$ the positive BE shift can be produced by several effects. XPS measurements of supported metal clusters and calculations on core-level binding energy shifts have been reported. In these studies several phenomena have been proposed to explain values up to $\sim 1 \mathrm{eV}$ in the binding energy shifts. ${ }^{46-50}$

The study of the valence-band spectra can yield valuable data on the behavior of metals toward the adsorption of different species, since the reactivity of transition metals is closely related to the population of the d-band. The closer the d-band center is to the Fermi level, the easier the charge transfer between the metal surface and the adsorbate states. Therefore, metals with d-bands populated near the Fermi level are capable of breaking bonds of the adsorbates on their surface. Figure 6 shows the

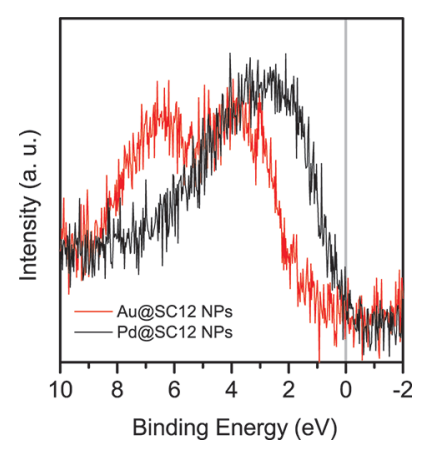

Figure 6. Valence-band signal for Pd@SC12 and Au@SC12 NPs. The intensity was normalized by the intensity of $\mathrm{Pd} 3 \mathrm{~d}$ and $\mathrm{Au} 4 \mathrm{f}$ signal, respectively.

valence-band spectra of Pd@SC12 and Au@SC12 NPs, measured with a $\mathrm{Mg} \mathrm{K} \alpha$ source. As it is observed, the valence band of Au NPs is located at greater binding energies than the one of Pd NPs. As already known in catalysis, this fact is closely related to the high reactivity of $\mathrm{Pd}$ compared to $\mathrm{Au}$. The changes in the density of states (DOS) of $\mathrm{Pd}(111)$ as a consequence of methanethiol adsorption were recently analyzed by means of density functional theory (DFT). ${ }^{1}$ It was proposed that the presence of sulfide species on $\mathrm{Pd}$ surfaces in contact with thiol molecules is produced due to the $\mathrm{S}-\mathrm{C}$ bond rupture by $\mathrm{Pd} .{ }^{1}$ Upon thiol adsorption on Pd, there is an electron density transfer from the metal d-band to the antibonding molecular orbitals of thiol molecules that weakens the $\mathrm{S}-\mathrm{C}$ bond, resulting in the elongation and finally breakage of $\mathrm{S}-\mathrm{C}$ bond. It was verified by DFT that after the adsorption of sulfide atoms on $\operatorname{Pd}(111)$, the surface is passivated, and the position of the d-band is shifted toward values more similar to the ones found on $\mathrm{Au}$, where it is known that $\mathrm{S}-\mathrm{C}$ bond scission does not occur for the case of alkanethiols. Once some sulfide is adsorbed, the surface cannot break more $\mathrm{S}-\mathrm{C}$ bonds, and accordingly, the thiols adsorption becomes possible on top of this diluted palladium sulfide layer. Cook et al. ${ }^{45}$ attributed a 
valence-band shift to a higher d-electron depletion on thiolateprotected Pd NPs compared to alkylamines-protected particles. This d-electron depletion is produced by the charge transfer from Pd to adsorbed sulfur species.

On the basis of the analysis of the above presented experimental data and the DFT results previously published, we present a plausible explanation for the processes that occur during the synthesis of dodecanethiolate-protected Pd NPs. In the case of place-exchange of dodecylamine by dodecanethiol, the processes should be very similar to the ones observed on extended planar Pd surfaces. Thiol molecules might replace alkyl amine molecules and approach the metallic $\mathrm{Pd}$, which is able to cleave the $\mathrm{S}-\mathrm{C}$ bond and produce sulfide adsorbates. At this point, the particle surface is not active anymore for the alkanethiol decomposition into sulfide, but it is able to adsorb dodecanethiolate moieties. Thus, the depletion of the population of valence-band electrons near the Fermi level explains why the Pd NPs are not completely sulfidized. Since Pd@NC12-SC12 NPs, prepared through the ligand-exchange strategy, showed the same sulfur species as those prepared following the two-phase Brust-Schiffrin method, it is reasonable to propose a similar mechanism for the final steps in the formation of Pd@SC12 NPs. This method starts with the addition of alkanethiol to the organic phase, which partially converts the $\mathrm{Pd}(\mathrm{II})$ halogenide complexes into $\mathrm{Pd}(\mathrm{II})$-thiolate complexes. Upon addition of the reducing agent, $\operatorname{Pd}(0)$ nuclei are formed. The small metallic particles grow in the presence of several species that can be adsorbed on its surface (tetraoctylammonium cations, halogenides, palladium thiolates, dodecanethiol, or species that are related with this thiol). Then, dodecanethiolate-related species might reach the metallic clusters, which are able to cleave the $\mathrm{S}-\mathrm{C}$ bond, and from this point the reaction should continue in the same way as in the other route. However, the synthesis does only drive to stable nanoparticle products if $\mathrm{Pd}(\mathrm{II})$ is in stochiometric excess with respect to the thiols (see UV-vis data in the Supporting Information and the results by Zamborini et al. ${ }^{24}$ ). Nevertheless, further studies are needed to completely elucidate each of the steps in the synthesis of thiolate-protected Pd NPs through the Brust-Schiffrin method, as the ones recently published for the case of $\mathrm{Au}, \mathrm{Ag}$, and $\mathrm{Cu}^{25,26,51}$

\section{CONCLUSIONS}

Even today, the chemistry of the thiolate-protected $\mathrm{Pd}$ nanoparticles produced either by the two-phase Brust-Schiffrin method or by the ligand place-exchange method is not well understood. On the basis of diverse experimental results, they have been described by different groups in terms of simple thiolate-capped $\operatorname{Pd}(0)$ particles (similar to thiolate-capped $\mathrm{Au}$ NPs), alkyldisulfide-capped $\operatorname{Pd}(0)$ particles, palladium sulfide particles, $\operatorname{Pd}(0)$ clusters immersed in a palladium sulfide phase, or complex thiolate-sulfide capped $\operatorname{Pd}(0)$ particles, in analogy to the surface structure reported for alkanethiolate SAMs on $\operatorname{Pd}(111)$. Our experimental data for $\sim 3 \mathrm{~nm}$ diameter Pd NPs prepared by both synthetic routes are consistent with the thiolate-sulfide capped $\operatorname{Pd}(0)$ particle composition. The NPs consist of a central core composed of metallic Pd, surrounded by a sulfidized $\mathrm{Pd}$ layer to which thiolate ligands are coordinated. Indeed, sulfur species in the Pd NPs should be present at the submonolayer level. In the present work we unambiguously demonstrate that the chemical nature of these particles is very similar to that experimentally found for alkanethiolate-covered bulk Pd and that recently proposed for $\operatorname{Pd}(111)$ surface, from DFT models.

The $\operatorname{Pd}(0)$ clusters, formed as a consequence of the reduction of $\mathrm{Pd}(\mathrm{II})$ species, are likely responsible for the S$\mathrm{C}$ bond cleavage that leads to adsorbed sulfide. The Pd cores modified by a submonolayer of sulfide are active for the adsorption of thiolate moieties, but they are not able to further decompose the thiol molecules. This result rules out the complete sulfidization of the Pd NPs, at least at this particle size. Then, the results from this paper are valuable not only for handling thiolate-protected Pd NPs for different applications but also for the basic comprehension of metallic nanoparticles and the relation of their surface structure with the synthesis method.

\section{ASSOCIATED CONTENT}

\section{S Supporting Information}

Synthesis of Pd and Au NPs, UV/vis study of the different steps in the synthesis of Pd@SC12 NPs, TEM of Au@SC12 NPs, FTIR details, and S 2p XPS signal of Pd@SC12 NPs acquired with synchrotron radiation. This material is available free of charge via the Internet at http://pubs.acs.org.

\section{AUTHOR INFORMATION}

\section{Corresponding Author}

*Fax: +54 221425 4642. Phone: +54 221425 7430. E-mail: mfonti@inifta.unlp.edu.ar. Homepage: http://nano.quimica. unlp.edu.ar.

\section{Notes}

The authors declare no competing financial interest.

\section{ACKNOWLEDGMENTS}

We thank S. Mejía-Rosales, M. Mariscal, and A. Ponce for useful discussions. We acknowledge financial support from Agencia Nacional de Promoción Científica y Tecnológica (PICT 2010-0423, PICT 2006-621, PICT 2010-2554, Nanotechnology Network PAE22711), CONICET (PIP 11220090100139), Universidad Nacional de La Plata, Argentina, Laboratório Nacional do Luz Síncrotron (LNLS), Campinas, Brazil (Research Proposal SGM-11769), The Welch Foundation Agency (project AX-1615: Controlling the Shape and Particles Using Wet Chemistry Methods and Its Application to Synthesis of Hollow Bimetallic Nanostructures), and the National Science Foundation (NSF) (PREM grant number DMR-0934218, NSF Grant 1103730: Alloys at the Nanoscale; The Case of Nanoparticles Second Phase). G.C. gratefully acknowledges the Swiss National Science Foundation (SNSF) and Universidad Nacional de La Plata for financial support.

\section{REFERENCES}

(1) Carro, P.; Corthey, G.; Rubert, A. A.; Benitez, G. A.; Fonticelli, M. H.; Salvarezza, R. C. Langmuir 2010, 26, 14655-14662.

(2) Vericat, C.; Vela, M. E.; Benitez, G.; Carro, P.; Salvarezza, R. C. Chem. Soc. Rev. 2010, 39, 1805-1834.

(3) Brust, M.; Walker, M.; Bethell, D.; Schiffrin, D. J.; Whyman, R. J. Chem. Soc., Chem. Commun. 1994, 801-802.

(4) Bourg, M.-C.; Badia, A.; Lennox, R. B. J. Phys. Chem. B 2000, 104, $6562-6567$.

(5) Jadzinsky, P. D.; Calero, G.; Ackerson, C. J.; Bushnell, D. A.; Kornberg, R. D. Science 2007, 318, 430-433.

(6) Templeton, A. C.; Wuelfing, W. P.; Murray, R. W. Acc. Chem. Res. 2000, 33, 27-36. 
(7) Love, J. C.; Wolfe, D. B.; Haasch, R.; Chabinyc, M. L.; Paul, K. E.; Whitesides, G. M.; Nuzzo, R. G. J. Am. Chem. Soc. 2003, 125, 25972609.

(8) Corthey, G.; Rubert, A. A.; Benitez, G. A.; Fonticelli, M. H.; Salvarezza, R. C. J. Phys. Chem. C 2009, 113, 6735-6742.

(9) Marshall, S. T.; Schwartz, D. K.; Medlin, J. W. Langmuir 2011, 27, 6731-6737.

(10) Marshall, S. T.; O’Brien, M.; Oetter, B.; Corpuz, A.; Richards, R. M.; Schwartz, D. K.; Medlin, J. W. Nat. Mat. 2010, 9, 853-858.

(11) Majumder, C. Langmuir 2008, 24, 10838-10842.

(12) Moreno, M.; Ibañez, F. J.; Jasinski, J. B.; Zamborini, F. P. J. Am. Chem. Soc. 2011, 133, 4389-4397.

(13) Sadeghmoghaddam, E.; Lam, C.; Choi, D.; Shon, Y.-S. J. Mater. Chem. 2010, 21, 307-312.

(14) Novakova, E. K.; McLaughlin, L.; Burch, R.; Crawford, P.; Griffin, K.; Hardacre, C.; Hu, P.; Rooney, D. W. J. Catal. 2007, 249, 93-101.

(15) Ramallo-López, J. M.; Giovanetti, L.; Craievich, A. F.; Vicentin, F. C.; Marín-Almazo, M.; José-Yacaman, M.; Requejo, F. G. Phys. B 2007, 389, 150-154.

(16) Zelakiewicz, B. S.; Lica, G. C.; Deacon, M. L.; Tong, Y. J. Am. Chem. Soc. 2004, 126, 10053-10058.

(17) Cargnello, M.; Wieder, N. L.; Canton, P.; Montini, T.; Giambastiani, G.; Benedetti, A.; Gorte, R. J.; Fornasiero, P. Chem. Mater. 2011, 23, 3961-3969.

(18) Lu, C.-H.; Chang, F.-C. ACS Catal. 2011, 1, 481-488.

(19) Litrán, R.; Sampedro, B.; Rojas, T. C.; Multigner, M.; SánchezLópez, J. C.; Crespo, P.; López-Cartes, C.; García, M. A.; Hernando, A.; Fernández, A. Phys. Rev. B 2006, 73, 054404.

(20) Walter, M.; Akola, J.; Lopez-Acevedo, O.; Jadzinsky, P. D.; Calero, G.; Ackerson, C. J.; Whetten, R. L.; Grönbeck, H.; Häkkinen, H. Proc. Natl. Acad. Sci. U. S. A. 2008, 105, 9157-9162.

(21) Leff, D. V.; Brandt, L.; Heath, J. R. Langmuir 1996, 12, 47234730.

(22) Yeh, J. J.; Lindau, I. At. Data Nucl. Data Tables 1985, 32, 1-155.

(23) Chen, S.; Huang, K.; Stearns, J. A. Chem. Mater. 2000, 12, 540547.

(24) Zamborini, F. P.; Gross, S. M.; Murray, R. W. Langmuir 2001, 17, 481-488.

(25) Li, Y.; Zaluzhna, O.; Xu, B.; Gao, Y.; Modest, J. M.; Tong, Y. J. J. Am. Chem. Soc. 2011, 133, 2092-2095.

(26) Goulet, P. J. G.; Lennox, R. B. J. Am. Chem. Soc. 2010, 132, 9582-9584.

(27) Yang, Z.; Klabunde, K. J.; Sorensen, C. M. J. Phys. Chem. C 2007, 111, 18143-18147.

(28) Yang, Z.; Smetana, A. B.; Sorensen, C. M.; Klabunde, K. J. Inorg. Chem. 2007, 46, 2427-2431.

(29) Ibanez, F. J.; Zamborini, F. P. J. Am. Chem. Soc. 2008, 130, 622633.

(30) Varela, M.; Lupini, A. R.; Benthem, K.; van; Borisevich, A. Y.; Chisholm, M. F.; Shibata, N.; Abe, E.; Pennycook, S. J. Annu. Rev. Mater. Res. 2005, 35, 539-569.

(31) Sun, Y.; Frenkel, A. I.; Isseroff, R.; Shonbrun, C.; Forman, M.; Shin, K.; Koga, T.; White, H.; Zhang, L.; Zhu, Y.; et al. Langmuir 2006, 22, 807-816.

(32) Liu, Y.; Wang, C.; Wei, Y.; Zhu, L.; Li, D.; Jiang, J. S.; Markovic, N. M.; Stamenkovic, V. R.; Sun, S. Nano Lett. 2011, 11, 1614-1617.

(33) Lu, W.; Wang, B.; Wang, K.; Wang, X.; Hou, J. G. Langmuir 2003, 19, 5887-5891.

(34) Hostetler, M. J.; Stokes, J. J.; Murray, R. W. Langmuir 1996, 12, 3604-3612.

(35) Manna, A.; Imae, T.; Yogo, T.; Aoi, K.; Okazaki, M. J. Colloid Interface Sci. 2002, 256, 297-303.

(36) Schaaff, T. G.; Shafigullin, M. N.; Khoury, J. T.; Vezmar, I.; Whetten, R. L. J. Phys. Chem. B 2001, 105, 8785-8796.

(37) Hayashi, M.; Shiro, Y.; Murata, H. Bull. Chem. Soc. Jpn. 1966, 39, $112-117$.

(38) Murayama, H.; Ichikuni, N.; Negishi, Y.; Nagata, T.; Tsukuda, T. Chem. Phys. Lett. 2003, 376, 26-32.
(39) Newville, M. J. Synchrotron Radiat. 2001, 8, 322-324.

(40) Ravel, B.; Newville, M. J. Synchrotron Radiat. 2005, 12, 537541.

(41) Ankudinov, A. L.; Ravel, B.; Rehr, J. J.; Conradson, S. D. Phys. Rev. B 1998, 58, 7565-7576.

(42) Zhong, C.-J.; Brush, R. C.; Anderegg, J.; Porter, M. D. Langmuir 1999, 15, 518-525.

(43) Shen, C. M.; Su, Y. K.; Yang, H. T.; Yang, T. Z.; Gao, H. J. Chem. Phys. Lett. 2003, 373, 39-45.

(44) Powel, C. J.; Jablonsky, A. NIST Electron Inelastic-Mean-FreePath Database, Version 1.2, SRD 71; National Institute of Standars and Technology: Gaithersburg, MD, 2010.

(45) Cook, S. C.; Padmos, J. D.; Zhang, P. J. Chem. Phys. 2008, 128, 154705-11.

(46) Wertheim, G. K.; DiCenzo, S. B.; Buchanan, D. N. E. Phys. Rev. B 1986, 33, 5384-5390.

(47) Wertheim, G. K.; DiCenzo, S. B. Phys. Rev. B 1988, 37, 844847.

(48) Mason, M. G. Phys. Rev. B 1983, 27, 748-762.

(49) Richter, B.; Kuhlenbeck, H.; Freund, H.-J.; Bagus, P. S. Phys. Rev. Lett. 2004, 93, 026805.

(50) Moriarty, P. Phys. Rev. Lett. 2004, 92, 109601.

(51) Li, Y.; Zaluzhna, O.; Tong, Y. J. Langmuir 2011, 27, 7366-7370. 